\title{
Analysis and Research of Online Measurement Technology in Inner Bore of EML
}

\author{
Ronggang Cao ${ }^{1+}$, Erwa Dong ${ }^{1}$ and Xiangcai Xu ${ }^{1}$ \\ ${ }^{1}$ Beijing Institute of Technology
}

\begin{abstract}
With the development of science and technology, as a new concept weapon electromagnetic gun has important strategic significance. In the process of launch, there are some damages including wear, corrosion, gouging and transition for the rail-gun. In order to study damage mechanism of the electromagnetic rail-gun during launch, it is necessary to detect the bore profile of electromagnetic rail-gun. The bore of rail-gun has the structural characteristics of closed, invisible and narrow space makes inconvenience for the measurement of the contour, so the online measurement and analysis of the rail-gun bore is very important. By comparing different methods of measurement, laser triangle method of noncontact is the heart of the way using in this paper. The laser triangle method has the characteristics of high precision and non-contact, and can realize on-line nondestructive measurement of arbitrary cross section of the rail bore. Through the comparison of the ideal contour and the measured profile of the electromagnetic rail-gun, the damage profile can be obtained. Using this bore profile scanner of electromagnetic rail-gun designed by this method, we can get two-dimensional profile images.
\end{abstract}

Keywords: Bore profile measurement, electromagnetic rail-gun, laser triangulation, nondestructive testing.

\section{Introduction}

Electromagnetic emission is a new way of transmitting objects to the higher speed by electromagnetic force. It converts electromagnetic energy into kinetic energy of payload. Based on this principle, it has incomparable advantages over other methods of transmission. Compared with the traditional way of transmitting, electromagnetic emission has the merits such as the higher initial velocity, higher precision, longer range, lower noise in emissions and smokeless. Electromagnetic rail-gun is an important research direction of electromagnetic emission, and has important application prospects.

Due to the strategic advantages of electromagnetic rail-gun, many countries want to go out of the laboratory into the application stage. But the electromagnetic emission affected by the transient current, strong magnetic field, high-speed sliding electrical contact and other adverse conditions, causing that track material is easily damaged under repeated emission, and seriously infect on the electromagnetic rail-gun combat-oriented. Thus It is particularly important that rail material is high temperature resistance, high yield strength and high hardness, that study insulation support mechanism of the design of the electromagnetic launcher ${ }^{[1]}$. Therefore, studying the damage and deformation mechanism of electromagnetic emission is important and urgent.

\section{Damage analysis}

The structure of the electromagnetic orbital launcher is shown in figure 1, the current through track and armature forming circuit. Because of the role of Lorents force, the rail from breech to armature generates mutual repulsion in this process. Due to a large amount of current flowing through the armature in a short time, the armature is heated and expanded, thus generating a squeeze force on both sides of the rail, and the

\footnotetext{
+ Corresponding author. Tel.: +86-1068918450.
}

E-mail address: crgeea@bit.edu.cn. 
extrusion pressure will decrease gradually due to armature wear. At the same time, the elastic fixing device of track provides a reaction force for track. In the electromagnetic launch process, there will be many kinds of damage in electromagnetic rail-gun.

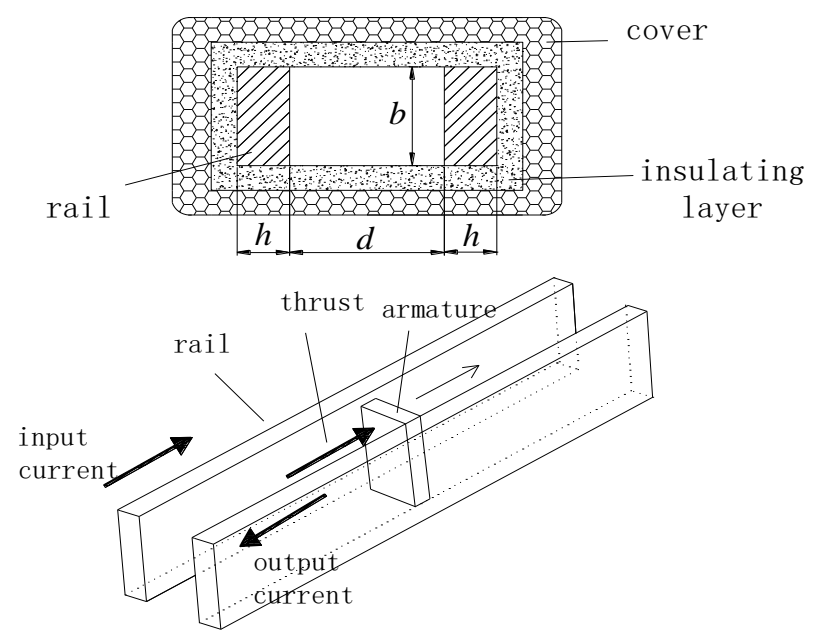

Fig. 1. The structure of the electromagnetic orbital launcher.

Through the analysis and research on electromagnetic rail-gun bore injury, the damage types have wear, corrosion, transition and planing. Groove is a kind of common damage in the course of electromagnetic railgun launch, it has an accumulated effect ${ }^{[2]}$. And the greater the incoming current, the more serious the damage will be. The slot etching pits will affect the electrical contact between the armature and the track after repeated firing of the electromagnetic rail-gun, which will damage the gun barrel and affect the performance of the electromagnetic rail-gun ${ }^{[3-5]}$.

The transition is another common phenomenon in the process of rail-gun launch ${ }^{[6]}$. During launch, the transient high temperature produced by large current will cause melt of contact part in the surface of the rail and armature. Before the armature arrive muzzle, between the rail and armature contact without cracks, there will be the phenomenon of transition. Meanwhile, the arc ablation produced by transition will seriously damage to the track ${ }^{[7]}$.

In the cause of electromagnetic rail-gun launch, there is a kind of planing phenomenon also. Because of the super high speed movement of the armature and the irregularity of the inner surface of the orbit, the slot is produced when the electromagnetic gun is fired. In the course of emission, the sliding process of the armature along the track shows a transient local high-intensity lateral loading process. The rail is subjected to frictional forces that move along the direction of motion and the normal preload and electromagnetic forces toward the outside of the interface perpendicular to the direction of motion. It is easy to produce shear effect on the rail surface, form planing damage, destroy the integrity of track and reduce track life. Gradually, dig depth along the launch direction first gradually become deep and quickly become shallow, and the finishing position will roll up some track material backward. A typical plane shape is like a graph ${ }^{[8]}$.

The deformation and damage of the electromagnetic launch seriously affect performance such as the lunching precision, orbital lifetime and repeated emission of electromagnetic rail-gun. In order to study the problem of orbit life in electromagnetic rail-gun launching technology, we need to measure changes in surface morphology before and after the launch of the rail, and analysis various phenomena characterized emission process, then put forward some preventive measures. During the each launch, various damage, produced by electrical contact between the armature and the rail armature high speed sliding in the rail when the pulse current passes, will left the different physical characteristics in the bore. Measurement and analysis of rail bore profile is an important means of analysis damage mechanism, solving damage problems and assessment lifetime of transmitter. Based on the above analysis, the electromagnetic gun will produce various damages to the inner bore of the track during the launching process. In order to detect the damage degree of the electromagnetic gun, a measuring device based on laser triangulation is proposed in this paper. 


\section{Principle of the device}

At present, in the field of electromagnetic emission technology, there are two types in the diagnosis of the bore contour, including commercial non online measurement device and on-line measuring device of mechanical contact. Non online measurement device's timeliness is on the poor, and it is a kind of non-realtime measurement. Because of restrictions by commercial profile measurements, it cannot enter the inner bore of the electromagnetic rail-gun, resulting that we need to disassemble the measurement of electromagnetic launcher in each measurement. By doing this, it is a permanent damage to the electromagnetic rail-gun, meanwhile the measurement cost is greatly increased and disassemble of gun barrel reduces the measurement efficiency. For mechanical contact profilometer, although it's the real-time online measurement, but contact measurement may cause some unnecessary damage to the orbital bore thereby affecting the measurement accuracy. Second, it relies on the contact pressure sensing, resulting in slow operation, long measurement time, and level of automation is not high actually. Due to the limitation of electromagnetic rail-gun interior, the design of its mechanical structure is easily restricted.

In order to study the damage formation mechanism of electromagnetic rail-gun in the course of launching, the measurement instrument for testing the bore of electromagnetic launcher, based on an image sensor, is developed in this paper. This method using in the instrument is a non-contact measurement method, outputting online 2D image bore profile. At the same time, the device is based on CMOS chip imaging, achieving triangular measurement data processing, using low-cost commercial CMOS chip which is low cost, easy to upgrade and expense system. The device solves the problems of the large scale range contour measurement of the bore and technical problems of 2D profile scanning for long and narrow track. It is of great significance to the development of electromagnetic rail-gun. The device can avoid the disassembly of the orbit emitter device on large range, realize the measurement of the change of the inner bore profile of the track after multiple emissions, reduce the measuring cost and increase the measuring efficiency. The device, that does not need to be disassemble, makes the electromagnetic rail-gun can achieve online detection, extremely convenience of study on the emission of electromagnetic rail-gun great convenience, and has great significance to the study of track damage caused by multiple launch.

As far as the scan of surface morphology, those devices can be divided into two categories, mechanical contact measurement and optical measurement.

Mechanical contact measurement. At present, the contact mechanical surface profile scanner uses the contact pressure sensor, through the directly contacting measurement of the bore surface to get the outline shape of the bore. The device, based on the retractable probe with mechanical contact, moves along the surface of the measured object, and then obtains the surface morphology information through the relative displacement measurement of the probe extension. The operation and measurement of the method require datum plane, and the existing commercial surface profile measuring instrument usually has the requirements of the measured size, the structure being complex, when using having more restrictive conditions.

Optical measurement. In the optical measurement of the bore profile, the laser triangulation method, using the PSD and laser as the basic components, was used to measure the 3D reconstruction system for micro pipes and deep holes ${ }^{[9]}$. For example, in pipeline measurement and traditional gun bore measurement, the system mainly consists of pipe robot, morphology detector and curvature detector. The morphology detector can collect the cross section information of the pipe inner wall, and calculate the position of the points in the local coordinate system. The curvature detector is responsible for the local geometric properties of the central axis of the tube hole at the sampling location of the detector, and completes the construction of the pipeline axis, which can realize the three-dimensional reconstruction of the inner surface of the pipeline.

\section{Device design}

This design uses non-contact laser triangulation principle ${ }^{[10-11]}$, with CMOS image sensor and laser transmitter as the basic components. The method can measure the target without changing the shape of the contour. Optical triangulation laser has high precision and non-contact characteristics, without two damage in the whole measurement process of track, at the same time can provide quantitative detection results to be detected, the bore any position of cross section diagram and 3D display etc.. With the rapid development of 
microelectronics and optics, tiny optical triangulation probe has developed, and has been used for nondestructive detection of piped bore.

The laser optical triangulation measuring system consists of optical triangle head, motion control, positioning system, data display and analysis system. The measurement principle of laser triangulation is shown in Figure 2, the defect of the measured surface can be represented by the change of the height of the inner surface relative to the datum line. When the laser beam irradiates the measured surface, the change of the surface height causes the position of the imaging point to move. The lens is imaged to the photodetector and the position of the spot is recorded on the image plane of the detector.

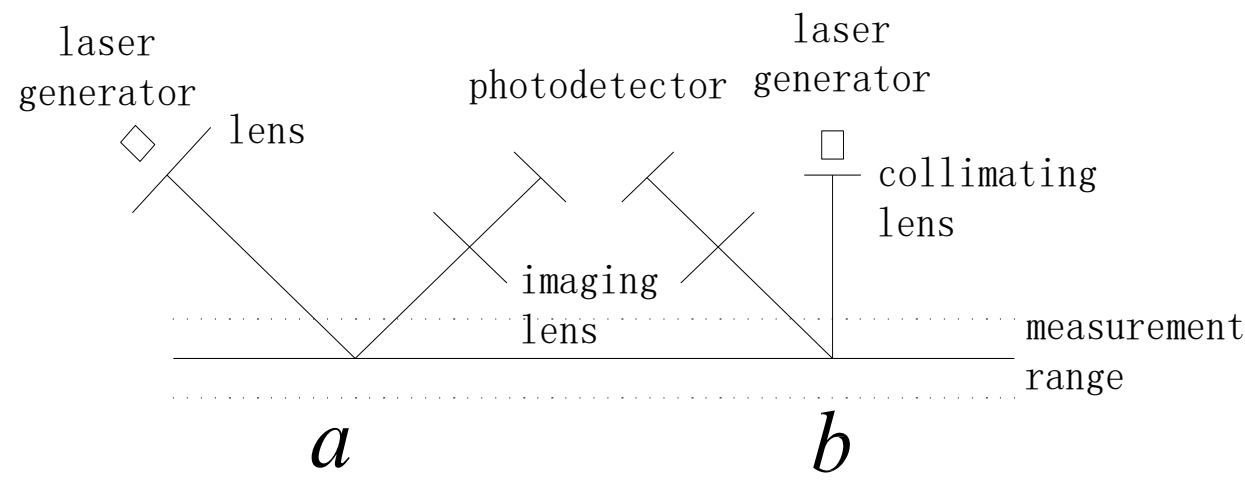

Fig. 2. The principle of the laser triangulation.

Figure $2 \mathrm{a}$ for detection of specular reflection surface, the use of photoelectric detectors to obtain the location of the reflected light to determine the surface defects; Figure $2 b$ for rough surfaces or surface detection with special reflections. The photodetector collects diffuse reflected light from the measured surface, and the laser is incident perpendicularly, light imaging of diffuse reflection of object surface by imaging lens. A photoelectric sensor placed on the image plane is used for receiving the imaging spot and converting the optical signal into an electrical signal. Because there is a direct link between the imaging position and the inner surface of the height, so the height of the surface can be achieved by calculating the surface defect detection.

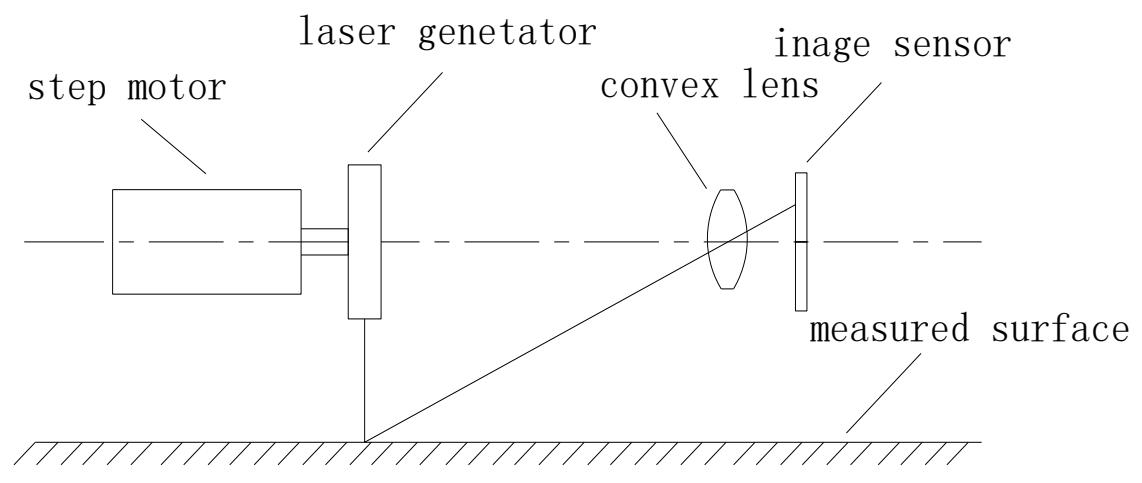

Fig. 3. The structure of the internal contour scanner.

The optical part should be designed to meet the requirements of a stable and reliable light source, which is an important prerequisite to ensure the accuracy of the system. The design is composed of a laser generator, a convex lens and an image sensor. The structure of the optical part is shown in Figure 3. This section determines the accuracy of the measurement results, requiring small laser beams and strong light. So choosing the appropriate light source has a great influence on the performance of the system. 


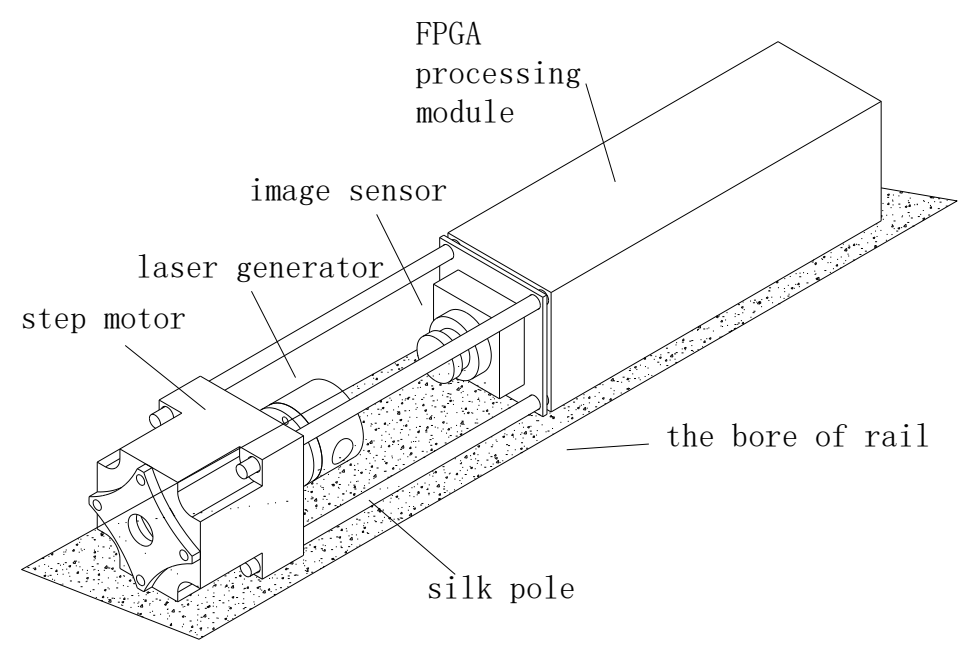

Fig. 4. The structure of the internal contour scanner.

Through the comprehensive comparison, this design measures the orbital bore surface using the method of Figure $2 b$ laser vertical incidence. And the structure of the inner scanner is shown in Figure 4 . In the experiment, the laser emits a beam of laser in the bore surface of electromagnetic rail-gun. The inner surface roughness will make the imaging position of different changes, image acquisition CMOS grasping the position of the light spot. Stepper motor every rotation angle, COMS image capture will grab a frame data. The image sensor outputs the frame data to FPGA for RGB565 data synthesis, then assigns a coordinate to the pixel order, through the calculation of the frame data is consistent with the judgment threshold. Finally, all the data which meet the threshold are integrated to get the contour of the bore.

After designing the device, we have a experiment for measurment the inner bore of rail, and through data processing, gained from the experiment, we can achieve the bore profile images that is several twodimensional profile shown in Figure 5. From comparision and analysis, we can get information about the level of damage in inner bore profile.

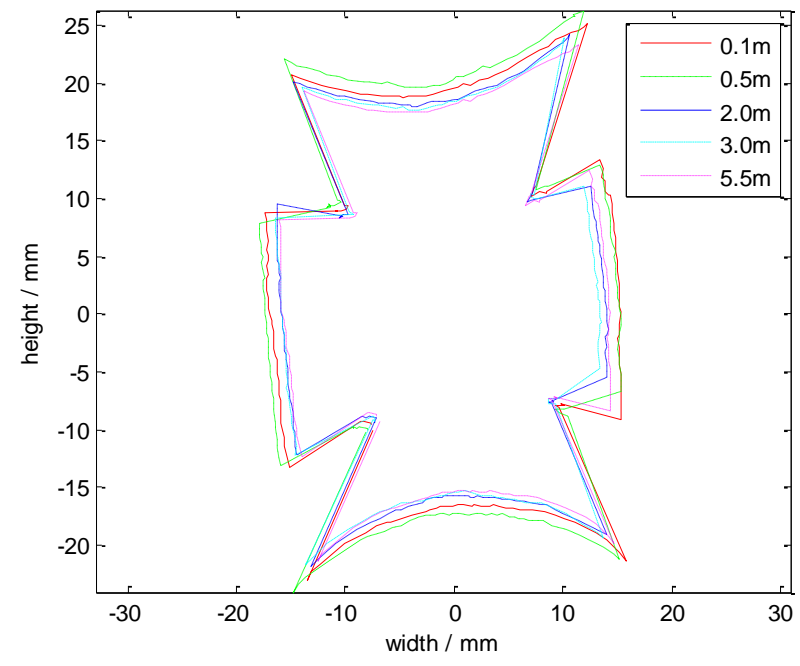

Fig. 5. The results of measurement.

\section{Conclusion}

In order to study the damage formation mechanism of the electromagnetic rail-gun in the course of launching better, an on-line measurement method based on laser triangulation is proposed for the measurement of orbit bore profile. By this method, we can solve the difficult problem of measuring the inner bore of the narrow orbit. At the same time, the method can also be applied to other devices for the measurement of the inner bore contour, which provides a feasible scheme for solving the similar problems. Although the method proposed in this paper has many advantages, there are still many deficiencies need to be improved. Because of the existence of four supporting rods in the whole process, there will be a dead 
corner, and the actual image is not complete. On the other hand, due to the different diameter of the orbital contour, the focal length of the camera is different, which results in the measurement of the contour of each orbit. But on the whole, the device is of great significance to the measurement of the inner hole of the electromagnetic rail-gun.

\section{Acknowledgements}

This work was supported by the National Natural Science Foundation of China (51407010)

\section{References (This is "Header 1" style)}

[1] Li Jun, Yan Ping, and Weiqun Yan, Electromagnetic Gun Technology and Its Development, High Voltage Engineering, vol.40, No. 4:1052-1064, April 30, 2014

[2] Watt T, Stefani F, Crawford M, et al. Investigation of damage to solid-armature railguns at startup, IEEE Transactions on Magnetics, vol.43 No1, 2007

[3] Baoyu Zhang and Huang wei, Grooving damage of rails under high-velocity sliding electrical contact, ORDNANCE MATERIAL SCIENCE AND ENGINEERING, vol.38 No.3, May, 2015

[4] K. P. Cooper, H. N. Jones, R. A. Meger, Analysis of railgun barrel materials, IEEE Transactions on Magnetics, vol.43 No.1:120-125, January, 2007

[5] Ronggang Cao, MingSu, Summarize and Analysis of Rail-gun's Grooving Phenomenon with Large Pulse Current, High Voltage Engineering, vol.42, No.9:2822-2829, September 30, 2016

[6] Guimin Liu, Zhongxu Yang, Yan Tao and Xiaoying Zhu, Current Status and Prospect on Rail Failures of Electromagnetic Railgun, Materials Review, vol.29, No.7:63-70, 2015

[7] Haiyao Cao and Zaiji Zhan, Experimental Study of Cu/Diamond Composite Electromagnetic Rail Ablation Characteristics, Chinese Journal of High Pressure Physics, vol.30, No.4:317-322, Aug, 2016

[8] Li He, Lei Bin, Zhiyuan Li, Qingao Lv and Zhang Qian, Analysis of Gouging Produced on Rail Surface of Electromagnetic Rail Gun, Journal of Gun Launch and Control, No.1:43-46, 2012

[9] WU En-qi, KE Ying-lin and LI Jiang-xiong, Photoelectric detection and 3D reconstruction system for the inner surface of small-diameter pipes, Opto-Electronic Engineering, vol.34, No.1, Jan,2007

[10] Richard D. Roberts, Laser Profilometry as an inspection method for Reformer Catalyst tubes, Materials Evaluation, vol.4, No.2:420 - 422, February, 1999

[11] Mccullough R W, Bondurant P D and Doyle J L. Laser-optical triangulations system provide new capabilities for remote inspection of interior surfaces. Materials Evaluation, 1338 - 1345, December, 1995 